\title{
Three-dimensional simulations of the atmosphere of an AGB star
}

\author{
B. Freytag ${ }^{1,2}$ and S. Höfner ${ }^{2}$ \\ 1 Centre de Recherche Astrophysique de Lyon, UMR 5574: CNRS, Université de Lyon, École Normale Supérieure de Lyon, \\ 46 allée d'Italie, 69364 Lyon Cedex 07, France \\ 2 Department of Physics and Astronomy, Division of Astronomy and Space Physics, Uppsala University, Box 515, 75120 Uppsala, \\ Sweden
}

Received 15 June 2007 / Accepted 27 February 2008

\section{ABSTRACT}

\begin{abstract}
Context. Winds of asymptotic giant branch stars are assumed to be driven by radiation pressure on dust. Previously, this process has been modeled with detailed time-dependent simulations of atmospheres and winds assuming spherically symmetric flows. In such models kinetic energy is injected by a variable inner boundary ("piston") simulating the effects of stellar pulsation. However, the dynamical processes in these atmospheres - convection and pulsations - are actually three-dimensional.

Aims. We present and analyze first 3D radiation hydrodynamics simulations of the convective interior and the atmosphere of a typical AGB star. In particular, we check whether the piston description in the 1D wind models is compatible with the 3D results.

Methods. We used two different RHD codes, one (CO5BOLD) to produce 3D models of the outer convective envelope and the inner atmosphere of an AGB star, the other to describe the atmosphere and the wind acceleration region, including dust formation and nongrey radiative transfer, but assuming spherically symmetric flows. From the movements of stellar surface layers in the 3D models, we derived a description for the variable inner boundary in the 1D models.

Results. The 3D models show large convection cells and pulsations that give rise to roughly spherically expanding shock waves in the atmosphere, levitating material into regions which are cool enough to allow for dust formation. The atmospheric velocity fields have amplitudes and time scales close to the values that are necessary to start dust formation in the 1D wind models.

Conclusions. The convection cells in the 3D simulations are so large that the associated shock fronts appear almost spherical, justifying the assumption of spherical symmetry and the use of a piston boundary condition in the context of wind models. Nevertheless, certain non-radial structures exist in the dust shell developing in the 3D simulations which should be detectable with current interferometric techniques.
\end{abstract}

Key words. convection - hydrodynamics - radiative transfer - stars: AGB and port-AGB - stars: atmospheres stars: winds, outflows

\section{Introduction}

Pulsation, dust formation, and mass loss through massive stellar winds are characteristic phenomena associated with asymptotic giant branch (AGB) stars. These processes play an important role in the evolution and for observable properties of both individual cool giants and stellar systems since AGB stars are major contributors to the chemical enrichment of the interstellar medium and to the integrated light of galaxies. Spectroscopic observations and models of nucleosynthesis indicate that AGB stars are important sources of carbon and sprocess elements, and possibly also of $\mathrm{Li}, \mathrm{N}$, and $\mathrm{F}$ (see, e.g., Lattanzio \& Wood 2004, for an overview). Quantitative predictions of yields are, however, still uncertain, mainly due to problems with modeling of convection and mass loss, two processes that are discussed in this paper.

Considerable theoretical and observational efforts have been made to understand dynamical atmospheres and winds of AGB stars and, in particular, the connection between fundamental stellar parameters and mass loss (see reviews by, e.g., Willson 2000; Woitke 2003; Höfner 2005; Höfner 2007). The basic picture of dynamical processes in atmospheres and winds of AGB stars can be summarized as follows: stellar pulsations cause waves which travel into the thinner outer layers of the atmosphere and develop into shocks with temperature and density variations strong enough to trigger intermittent dust formation. Large opacities allow the dust grains to capture the stellar radiation efficiently, leading to an outward acceleration of the dust particles which is transmitted to the gas by collisions, causing a slow but dense outflow from the star ${ }^{1}$.

Building on the results of pioneering models (e.g., Wood 1979; Bowen 1988) major progress has been made in recent years regarding numerical simulations of dynamical atmospheres and winds of cool giants. Important steps towards more realistic models include the introduction of detailed nonequilibrium descriptions of dust formation (e.g., Fleischer et al. 1992; Höfner \& Dorfi 1997; Jeong et al. 2003), and frequencydependent radiative transfer (e.g., Höfner et al. 2003; Woitke 2006b) in time-dependent dynamical simulations featuring effects of stellar pulsation and shock waves. Such models, however, usually rely on two basic ingredients, (i) the assumption of purely radial pulsations, leading to strictly spherically symmetric flows, which reduces the computational effort considerably

\footnotetext{
1 While this scenario seems to work nicely for C-rich AGB stars, it should be noted that recent work indicates serious problems for the standard picture in the case of M-type giants (Woitke 2006b; Höfner \& Andersen 2007). Frequency-dependent dynamical models predict that silicate grains forming in atmospheres of AGB stars will have an extremely low iron content, leading to opacities insufficient to drive a stellar wind. This problem was not apparent in earlier grey wind models (e.g., Jeong et al. 2003).
} 
compared to 3D simulations, and (ii) treating stellar pulsation as external input, typically in the form of variable inner boundary conditions just below the stellar surface ("piston models").

In this paper we discuss the validity of these two basic assumptions inherent to current AGB wind models. We use CO5BOLD to perform 3D radiation hydrodynamic "star-in-abox" simulations of the convective interior and the atmosphere of an AGB star to investigate the influence of spatial inhomogeneities induced by huge convection cells. As 3D calculations are much more time-consuming than 1D simulations, they have to rely on simpler physics (using grey opacities, not including radiation pressure at present) and they comprise a smaller spatial range, i.e. the convective outer envelope (excluding the stellar core) and the immediate surroundings of the star. Nevertheless, these preliminary results give a first impression of the effects of non-spherical flows on the formation of dust grains.

The 3D models presented in this paper are complementary to the approach chosen by Woitke (2006a), who computed 2D (axisymmetric) dust-driven wind models, including time-dependent dust formation and grey radiative transfer, but excluding the central region containing the star itself. The purpose of these models was to study how intrinsic instabilities in the dust formation process create complex patterns in the circumstellar envelope, without taking the pulsation or convective motions of the central star into consideration.

Multi-dimensional simulations of stellar surface convection that account for realistic microphysics started with models of small patches of solar surface convection (Nordlund 1982), and are now performed by a number of groups (e.g., Steffen et al. 1989; Asplund et al. 2000; Skartlien et al. 2000; Stein \& Nordlund 2000; Gadun et al. 2000; Robinson et al. 2003; Vögler 2004; Wedemeyer et al. 2004). Simulations have been extended to patches or slices of red subgiants and giants (Asida \& Tuchman 1997; Asida 2000; Freytag \& Salaris 1999; Robinson et al. 2004; Collet et al. 2006), and even to entire AGB stars or red supergiants (see Jacobs et al. 1998; Freytag et al. 2002). Woodward et al. (2003) presented models with extremely high numerical resolution but simple microphysics.

In the current paper we present the first 3D models of the convective outer envelope and the atmosphere of an AGB star including the regions where dust forms. We demonstrate how the models can be used to derive a description of the dynamical inner boundary conditions required in 1D wind simulations and we compare selected 1D models using classical sinusoidal piston motion with the new description.

In Sect. 2 we present the numerical methods used in the 3D and $1 \mathrm{D}$ radiation hydrodynamics codes. Section 3 describes the setup and the results of the 3D simulations. In Sect. 4 we outline how we extract from the 3D models a formula for the piston motion to be used in the 1D models. The resulting 1D wind models are presented in Sect. 5. Section 6 summarizes our conclusions.

\section{Numerical methods}

Basic features of the two codes used here are compared in Table 1.

\subsection{D simulations with CO5BOLD}

The RHD code CO5BOLD (Freytag et al. 2002; Freytag et al. 2004) has been used to produce time-dependent 3D models of the outer convective layers and the atmosphere of an AGB star.

The code solves the coupled equations of compressible hydrodynamics and non-local radiation transport on a Cartesian grid with a time-explicit scheme. In the models presented here, the entire computational domain and the individual grid cells are simple cubes.

Gravitation is included as an external potential, with a general $1 / r$ profile that is smoothed in the central region of the star. In this volume, heat is added as a constant source term according to the desired stellar luminosity and a drag force is applied to damp flows through this artificial core. Without this force, strong global dipolar flows would occur (that oscillate and thus differ from the stationary dipolar flows found by Jacobs et al. 1998; Woodward et al. 2003). In contrast to the piston boundary of the 1D simulations described in Sect. 2.2 the "inner boundary condition" in the 3D models does not actively impose any dynamics. The outer boundaries (all six sides of the box) are open for matter and radiation.

The hydrodynamics scheme uses directional splitting and a 1D approximate Riemann solver of Roe-type, modified to account for the effects of ionization (by a tabulated equation of state) and the external gravitational potential. The optional use of an additional 3D tensor viscosity improves the stability. It is activated in the presented models.

The non-local radiative energy transfer is solved with a short characteristics scheme. It is conservative in the sense that the energy change per cell and time step can be derived from a discrete divergence of the radiative flux. All presented models have grey tabulated opacities, treat scattering as true absorption, and assume strict local thermodynamic equilibrium. CO5BOLD is also capable of performing "classical" local simulations of, e.g., granulation in small patches of the solar surface, using a constant gravity, periodic side boundary conditions, and a different radiation transport module (for instance, Aufdenberg et al. 2005; Wedemeyer-Böhm et al. 2005).

Efforts are underway to fully implement the dust model from the 1D RHD code presented in Sect. 2.2.1 into the 3D code. The basic processes of grain formation, destruction, and passive advection of dust are already included in the models presented here. The coupling of the grains to the radiation field, however, is still missing (no radiative pressure) which prohibits the formation of dust-driven winds.

\section{2. $1 D R H D$ simulations}

The frequency-dependent 1D RHD models for atmospheres and winds of AGB stars have been described in detail by Höfner et al. (2003), and we only give a short summary of the main physical and numerical features here. These models allow us to compute wind properties like the mean outflow velocity, the dustto-gas ratio, and the mass loss rate. Synthetic spectra based on snapshots of the structures compare well with observations (e.g., Gautschy-Loidl et al. 2004; Nowotny et al. 2005a; Nowotny et al. 2005b).

The models cover a spherical shell with an inner boundary below the stellar photosphere but above the driving zone of stellar pulsations. The effects of pulsations (or, in the present case, large-scale convective motions) are simulated by temporal variations at the inner boundary. The location of the outer boundary depends on the dynamics of the model. The boundary conditions applied in this study are described in more detail in Sect. 5.

The variable structure of the atmosphere and wind is determined by the equations of hydrodynamics (equation of continuity, equation of motion, and energy equation) together with the frequency-integrated zeroth and first moment equations of radiative transfer (accounting for the energy and momentum balance of the radiative field). In the region covered by the models 
Table 1. Comparison of the two codes as they are used in the current work.

\begin{tabular}{lll}
\hline \hline Feature & 3D RHD (CO5BOLD) & 1D RHD \\
\hline Grid & 3D fixed Cartesian & 1D adaptive spherical \\
Region & outer convection zone and photosphere & photosphere and wind formation region \\
Time integration & explicit & implicit \\
Hydrodynamics & compressible, incl. ionization & compressible \\
Radiation & non-local & non-local \\
& short characteristics & long characteristics + Eddington tensors \\
Radiation pressure & no & yes \\
Opacity & grey & sampling $(64$ wavelength points $)$ \\
Gravity & prescribed potential & via Poisson equation \\
Dust & 4-moment formalism & 4-moment formalism, full coupling \\
Inner boundary & constant heating & piston $($ variable membrane $)$ \\
Outer boundary & open & open \\
CPU time per model & months $(8 \times 900 \mathrm{MHz}$ SunFire $)$ & hours $(1 \times 900 \mathrm{MHz}$ SunFire $)$ \\
\hline
\end{tabular}

energy transport is dominated by radiation and, consequently, none of the commonly used parameterized description of convective energy transport is applied when modeling the thermal structure of the atmosphere. A set of equations describing the time-dependent formation, growth, and evaporation of dust grains is solved simultaneously with the radiationhydrodynamical equations (see Sect. 2.2.1). The full set of RHD and dust equations is listed in Höfner et al. (2003).

The different frequency means of opacities as well as the Eddington factor which appear in the radiation-hydrodynamical equations require the knowledge of the frequency-dependent radiation intensity. The latter is obtained by solving the frequencydependent equation of radiative transfer for the current densitytemperature structure at each time step using the method of long characteristics. The models presented here are based on opacity sampling data of molecular line opacities for $\mathrm{CO}, \mathrm{CH}, \mathrm{C}_{2}$, $\mathrm{CN}, \mathrm{C}_{2} \mathrm{H}_{2}, \mathrm{HCN}, \mathrm{C}_{3}, \mathrm{TiO}, \mathrm{H}_{2} \mathrm{O}$, and $\mathrm{SiO}$, complemented by relevant continuous gas opacities (free-free transitions and scattering). For models cool enough to form dust-driven winds atomic line opacities play a minor role compared to molecules and dust as far as the structure of the atmosphere is concerned, and are therefore neglected in the current models. The radiative transfer equation is solved at 64 frequency points between 0.25 and $25 \mu \mathrm{m}$, which are selected considering the overall flux distribution of cool stars (equidistant in wavenumber within several subintervals), but randomly located with regard to opacity features.

The conservation laws for gas, dust, and radiation are solved implicitly using a Newton-Raphson scheme. The main features of the numerical technique used in the 1D model calculations are the following: a conservative (volume-integrated) formulation of the discretized radiation-hydrodynamics and dust equations, a monotonic second order advection scheme (van Leer 1977), as well as an adaptive grid. A so-called grid equation is solved simultaneously with the physical conservation equations and distributes the grid points according to accuracy considerations (Dorfi \& Drury 1987).

\subsubsection{Dust formation and destruction}

The models presented here include a time-dependent description of dust grain growth and evaporation using a method developed by Gail \& Sedlmayr (1988) and Gauger et al. (1990). In this approach, the dust component is described in terms of moments $K_{j}$ of the grain size distribution function weighted with a power $j$ of the grain radius. The moment $K_{0}$ represents the total number density of grains (integral of the size distribution function over all grain sizes) while the ratio $K_{3} / K_{0}$ is proportional to the average volume of the grains. If these moments (and thus the amount of material condensed into grains) are known, the various frequency-integrated opacities entering the RHD equations can be computed.

We solve the equations which determine the evolution of the dust component together with the radiation-hydrodynamics system described above, considering spherical grains consisting of amorphous carbon. The nucleation, growth, and evaporation of grains is assumed to proceed by reactions involving $\mathrm{C}, \mathrm{C}_{2}, \mathrm{C}_{2} \mathrm{H}$, and $\mathrm{C}_{2} \mathrm{H}_{2}$. The dust opacities are calculated using the data of Rouleau \& Martin (1991).

\section{3D models: stellar interior and atmosphere}

\subsection{Parameters and modeling procedure}

The stellar parameters of the 3D models presented here were chosen to closely resemble those of a 1D model studied by Höfner et al. (2003), i.e., $M=1 M_{\odot}, L=7000 L_{\odot}, R \sim 350 R_{\odot}$, $T_{\text {eff }} \sim 2800 \mathrm{~K}, \log g \sim-0.6$ (cgs units). As far as the description of the dust component is concerned (chemistry and optical properties of grains), both the $1 \mathrm{D}$ and 3D models assume a value of $\mathrm{C} / \mathrm{O}=1.4$. There are, however, differences concerning the gas opacities and the equation of state. While the $1 \mathrm{D}$ models assume a simple perfect gas law, the $3 \mathrm{D}$ models use a realistic equation of state (including ionization of $\mathrm{H}$ and $\mathrm{He}$ ) for a standard solar composition (with number fractions of $\mathrm{He}=0.09085$ and a representative metal $Z=0.00064)^{2}$. Regarding gas opacities, the $1 \mathrm{D}$ models use frequency-dependent molecular opacities corresponding to a $\mathrm{C}$-rich mixture $(\mathrm{C} / \mathrm{O}=1.4$; see Sect. 2.2 for details), while the 3D models are based on grey opacities for a solar composition $^{3}$. The grey gas opacity tables used in the 3D models have been merged at around $12000 \mathrm{~K}$ from high-temperature OPAL data (Iglesias et al. 1992) and low-temperature PHOENIX data (Hauschildt et al. 1997) by Hans-Günter Ludwig.

The 3D simulations start with a hydrostatic, spherically symmetric structure, mapped onto a 3D grid, adding small-amplitude

\footnotetext{
2 The slight inconsistency between the element abundances in the EOS and the dust chemistry in the $3 \mathrm{D}$ models has no noticeable effect in view of the relatively small contribution of metals to, e.g., the heat capacity. ${ }^{3}$ In this context, the inconsistency in the element abundances is probably secondary to the effects caused by the frequency-dependent vs. grey treatment of radiative transfer as far as the optically thin outer layers are concerned. In the inner parts of the model which are relevant for convection, however, both non-grey and abundance effects should be minor.
} 
Table 2. Basic parameters of the 3D models.

\begin{tabular}{llllllcc|ccc|l}
\hline \hline Label & Model & Grid & $\begin{array}{c}\text { Box } \\
{\left[R_{\odot}\right]}\end{array}$ & $\begin{array}{c}M_{\text {pot }} \\
{\left[M_{\odot}\right]}\end{array}$ & $\begin{array}{c}M_{\text {env }} \\
{\left[M_{\odot}\right]}\end{array}$ & $\begin{array}{c}L \\
{\left[L_{\odot}\right]}\end{array}$ & $\begin{array}{c}t \\
{[\mathrm{yr}]}\end{array}$ & $\begin{array}{c}R \\
{\left[R_{\odot}\right]}\end{array}$ & $\begin{array}{c}T_{\text {eff }} \\
{[\mathrm{K}]}\end{array}$ & $\begin{array}{l}\log g \\
(\mathrm{cgs})\end{array}$ & Comment \\
\hline \multirow{3}{*}{$\mathrm{S}$} & st28gm06n01 & $127^{3}$ & 1244 & 1 & 0.062 & $7000 / 6742$ & $0.0-5.4$ & 357 & 2768 & -0.67 & start from static sphere \\
& st28gm06n02 & $127^{3}$ & 1244 & 1 & 0.196 & $7000 / 7079$ & $0.0-14.2$ & 437 & 2531 & -0.85 & long sequence \\
L & st28gm06n03 & $171^{3}$ & 1674 & 1 & 0.189 & $7000 / 6589$ & $0.0-4.0$ & 400 & 2600 & -0.77 & extended box \\
L & st28gm06n05 & $171^{3}$ & 1674 & 1 & 0.186 & $7000 / 8144$ & $3.4-5.9$ & 423 & 2665 & -0.82 & extended, dust \\
st28gm06n06 & $171^{3}$ & 1674 & 1 & 0.186 & $7000 / 6935$ & $5.9-10.4$ & 429 & 2542 & -0.83 & extended, dust, new EOS \\
\hline
\end{tabular}

random velocities. In a sequence of consecutive model runs the physical parameters of the dynamical model and the size of the computational box are adjusted, and the development of convection is followed until the flow properties stabilize. Then, in the last run, dust formation is included as an add-on feature, without feeding the resulting dust opacities back into the RHD equations.

The details of this process, including the construction of the initial model, as well as physical and numerical challenges, are described in Appendix A. The relevant parameters of the 3D model runs are listed in Table 2. It shows labels for the small and the large 3D models used in this paper; the original model name (made from the first two digits of the approximate effective temperature, the approximate surface gravity, and a running number: for reference, e.g., to corresponding movies); the number of grid points; the edge length of the computational cube; the mass used to compute the potential; the total mass actually included in the model (the rest is assumed to sit in the unresolved stellar core); the luminosity (inserted into the core and measured over a certain interval); the start and end time of the simulation; the approximate radius, effective temperature, and surface gravity; and finally a comment.

Two sequences, labeled "S" ("small") and "L" ("large", the latter actually consisting of two runs) are discussed below. Model st28gm06n02 (denoted as "S") comprises a longer time sequence but does not reach as far out as the spatially more extended models st28gm06n05/06 (merged into model "L"), which also include terms that describe the formation and transport of dust.

\subsection{Results of the $3 D$ simulations}

To capture the essential properties of the complex convective flows and atmospheric shock waves of the 3D models we use different types of plots: temporal sequences of slices through the center of the computational box showing the evolution of densities, temperatures, and velocities (model S: Fig. 1, Cols. 1-3, Col. 4: surface intensities; model L: Fig. 2, Cols. 1-3, Col. 4: dust-to-gas ratio), and sets of spatial slices at different distances from the center of the box for a selected snapshot, illustrating the 3D structures (Fig. 3, model L). Quantitative data are plotted in Fig. 4.

The time sequence of grey (frequency-integrated) surface intensities of model S in Fig. 1 (Col. 4) shows a pulsating stellar atmosphere with complex large-scale structures. Even without the inclusion of the dust in the opacity tables the optical thickness of the atmosphere is so large that the top of the convective envelope becomes only occasionally visible (e.g., in snapshot at $t=12.37 \mathrm{yrs}$ in Fig. 1). At other times, shocks in the atmosphere cause the visible structures.

\subsubsection{Convection and pulsation}

Due to a peak in the opacities the temperature drops rapidly in the range from $11000 \mathrm{~K}$ to $3000 \mathrm{~K}$ with a corresponding decrease in entropy and increase in density. This leads to a density inversion at the distorted stellar surface that is clearly visible in Figs. 1 to 3 and that indicates the main region for convective instability. The layers where the temperature drops mark the top of the convection zone.

The star shows huge global convective structures. While individual elements are difficult to distinguish and therefore to count, there might typically be only two or three dominant cells present with lifetimes of years. The surface "granules" do not show up in the intensity images due to the large optical thickness of the layers above. However, e.g., comparing isothermes of $6000 \mathrm{~K}$ and the flow motions show the common pattern of granules with upflowing material surrounded by intergranular lanes. These start to turn into more finger-like structures at greater depths (at an iso-surface of about $20000 \mathrm{~K}$ ). However, the flows encounter the artificial stellar core before the transition is complete. At the surface the borders of the large cells are uneven, and often small short-lived cells show up.

At first glance, the size of the convective cells might come as a surprise. However, Schwarzschild (1975) argued with scaling properties of solar granules to estimate that only a small number of cells exists on the surface of a red supergiant. Solar granulation has characteristic (horizontal) scales of about 1000 to $2000 \mathrm{~km}$ while the photospheric (vertical) pressure scale height is about $150 \mathrm{~km}$. The last column in Fig. 4 shows the "hydrostatic" pressure scale height $\left(H_{\mathrm{p}}=P / g \rho\right)$ and the "actual" pressure scale height $\left(H_{\mathrm{p} \text {,dyn }}=(\operatorname{dlog} P / \mathrm{d} z)^{-1}\right)$ in solar radii and relative to the distance of each spherical layer to the stellar center. Nowhere does $H_{\mathrm{p} \text {,dyn }} / r$ drop below $10 \%$. And assuming the same scaling factor as in the Sun convective cells with an extent comparable to the stellar radius are to be expected. In addition, the strong coupling between gas and the radiation field in AGB stars tends to smooth small-scale temperature fluctuations and to favor larger cells.

With convective flows on global scales as encountered here, it becomes difficult to identify stellar pulsations in the classical sense of the word and, in particular, to distinguish "modes" which have been defined in the context of small-amplitude deviations from hydrostatic equilibrium. It seems, however, fair to say that the 3D models are pulsating, in the sense that the volume occupied by a major fraction of the mass (located below the stellar surface) is expanding and contracting more or less periodically on a typical time scale of a year. It may be futile to discuss whether these pulsations should be classified as radial or non-radial, since they combine changes in volume with changes in shape. Nevertheless, in Sect. 4 we derive a spherical analogue of these global expansions and contractions, in order to use it as an alternative description of stellar pulsation in the spherically symmetric wind models presented in Sect. 5 . 
$11.11 \mathrm{yrs}$
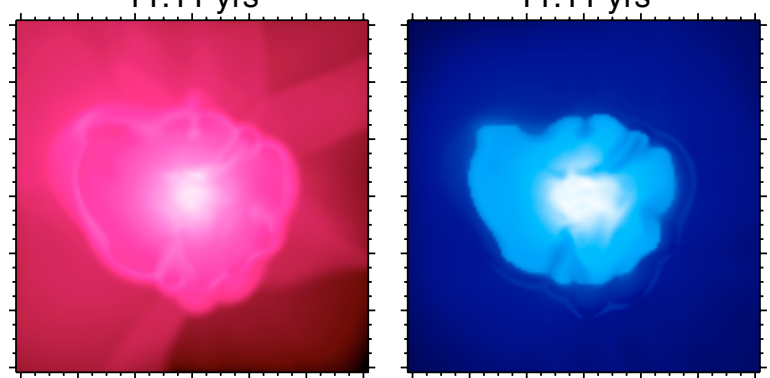

$\begin{array}{rrr}600-400-200 & 0 & 200 \\ 11.42 & \text { yrs }\end{array}$
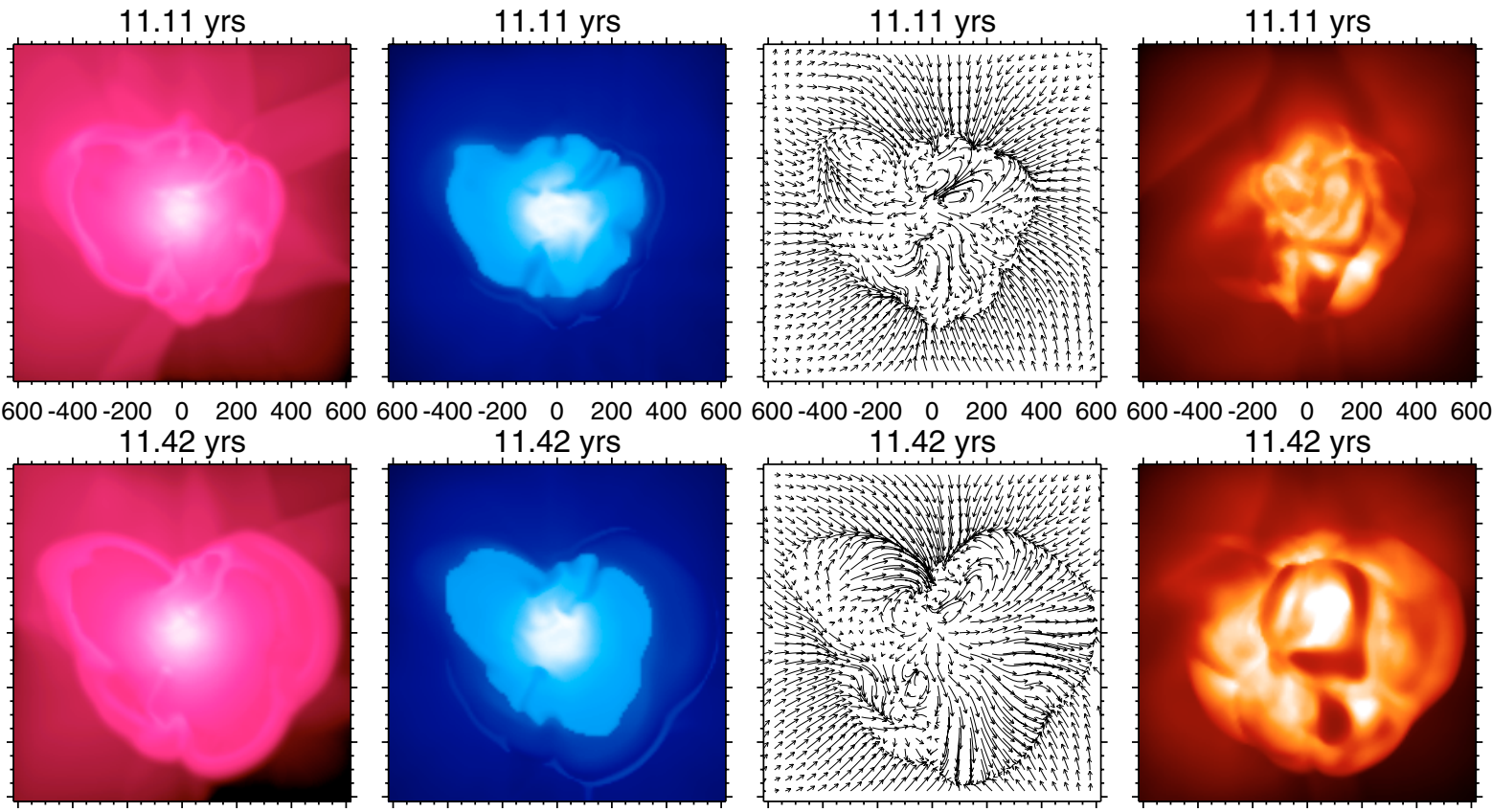

11.11 yrs

$600-400-200 \quad 0 \quad 200400600600-400-200 \quad 0 \quad 200400600600-400-200 \quad 0 \quad 200400600600-400-200 \quad 0 \quad 200400600$ $11.74 \mathrm{yrs}$
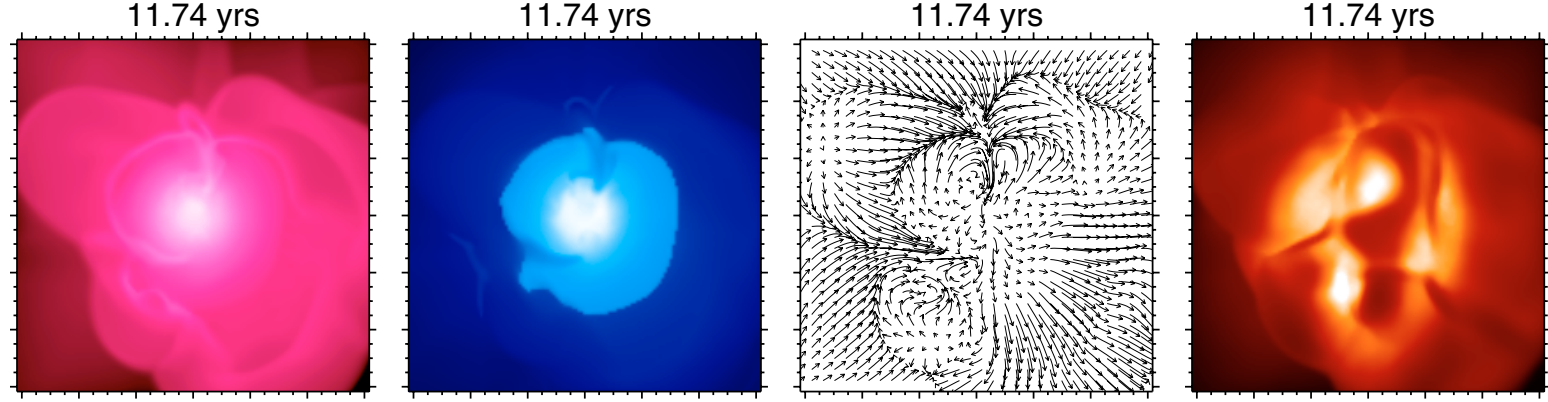

$600-400-200 \quad 0 \quad 200400600600-400-200 \quad 0 \quad 200400600600-400-200 \quad 0 \quad 200400600600-400-200 \quad 0 \quad 200400600$ 12.06 yrs

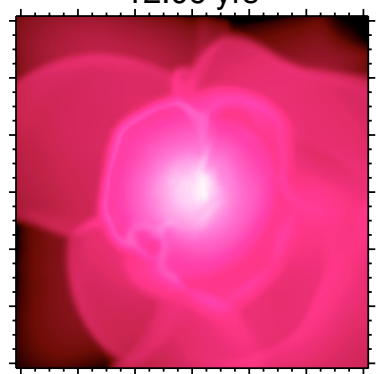
12.06 yrs

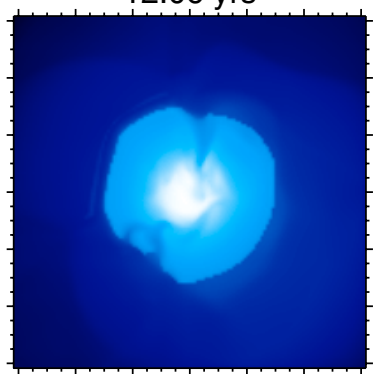
12.06 yrs
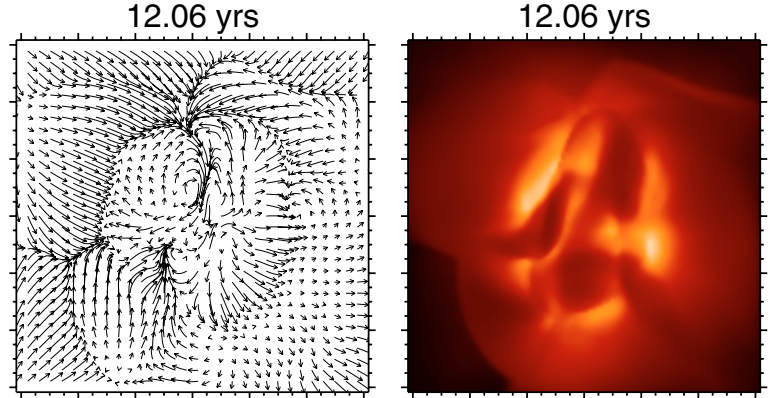

$600-400-200 \quad 0 \quad 200400600600-400-200 \quad 0 \quad 200400600600-400-200 \quad 0 \quad 200400600600-400-200 \quad 0 \quad 200400600$ 12.37 yrs
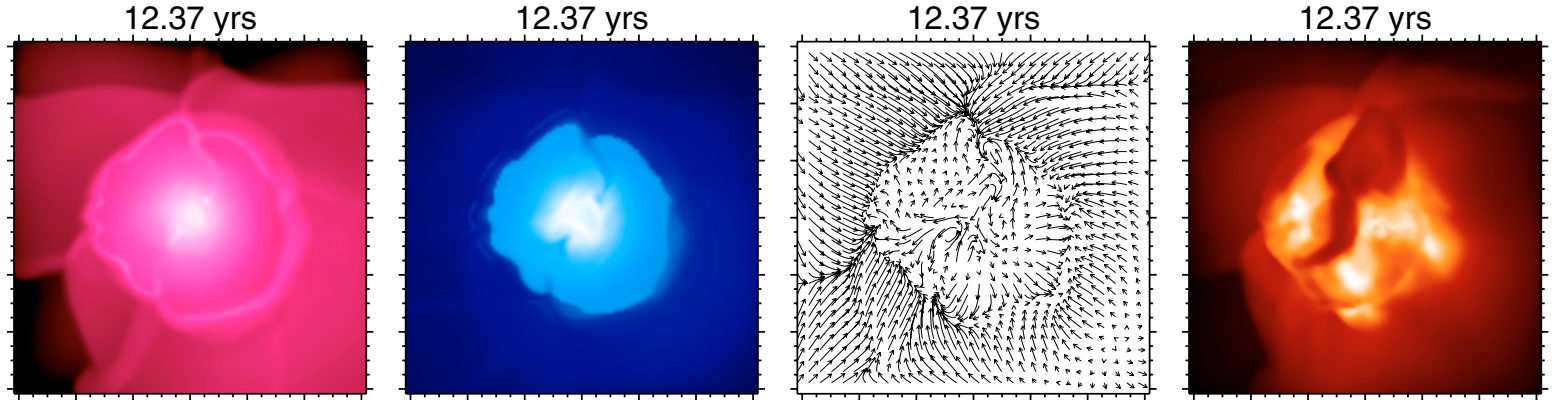

$600-400-200 \quad 0 \quad 200400600600-400-200 \quad 0 \quad 200400600600-400-200 \quad 0 \quad 200400600600-400-200 \quad 0 \quad 200400600$

Fig. 1. Time sequence of five snapshots for model S (st28gm06n02). The model age in years is indicated on top of each frame. The axes are in solar radii. Shown from left to right are log density (color range from $10^{-13}$ to $2 \times 10^{-7} \mathrm{~g} / \mathrm{cm}^{3}$ ), log temperature (color range from $800 \mathrm{~K}$ to $50000 \mathrm{~K}$ ), velocity field (pseudo-streamlines of the velocity components within the image plane integrated over $5 \times 10^{6} \mathrm{~s}$ ) and grey intensity. 

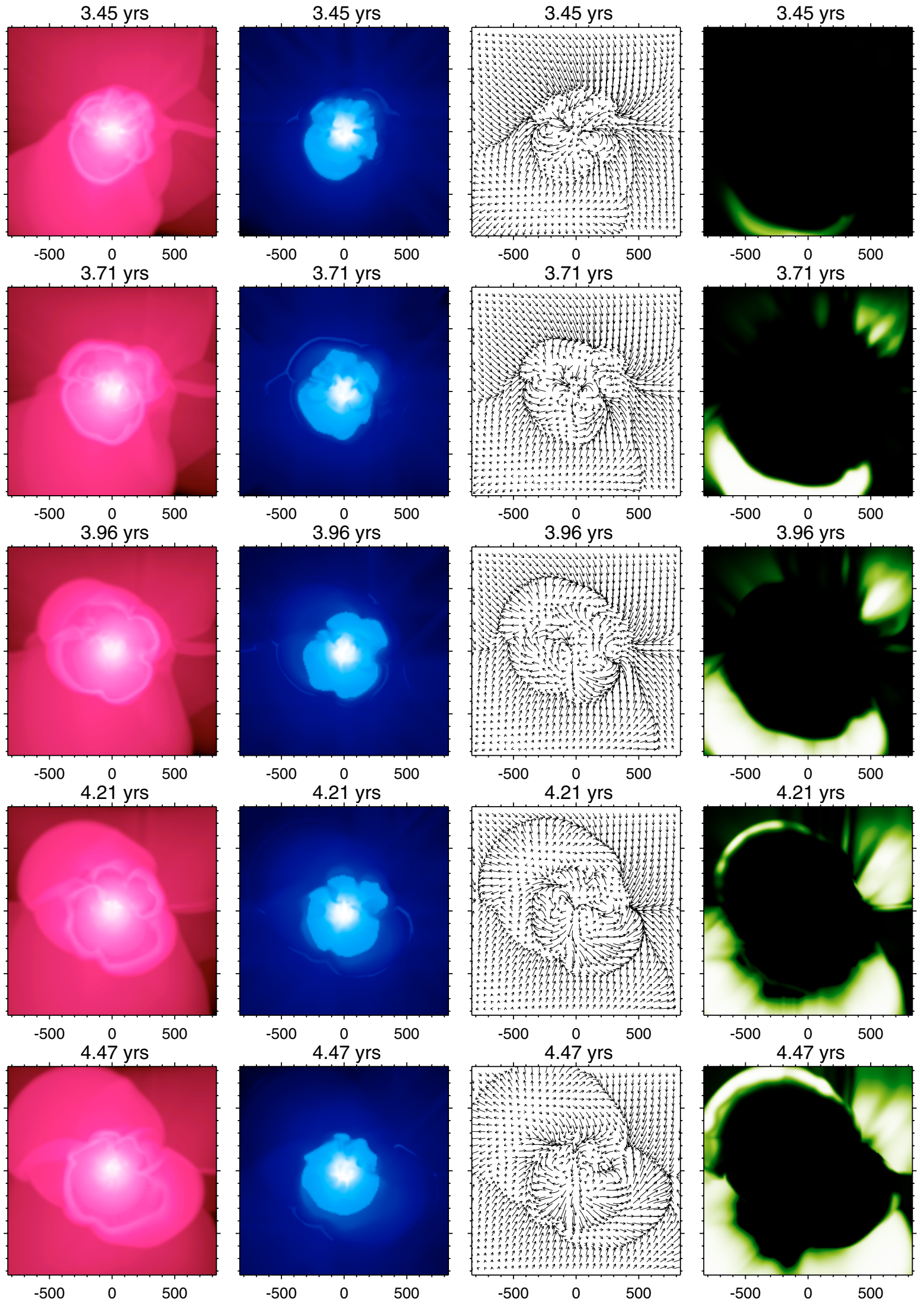

Fig. 2. Time sequence of five snapshots for model L (st28gm06n05). The model age in years is indicated on top of each frame. Axes are in solar radii. Shown from left to right are log density (color range from $10^{-16}$ to $2 \times 10^{-7} \mathrm{~g} / \mathrm{cm}^{3}$ ), log temperature (color range from $800 \mathrm{~K}$ to $50000 \mathrm{~K}$ ), velocity field (pseudo-streamlines of the velocity components within the image plane integrated over $5 \times 10^{6} \mathrm{~s}$ ), and degree of dust condensation (ranging from 0 to 1 , with black indicating dust-free regions). 

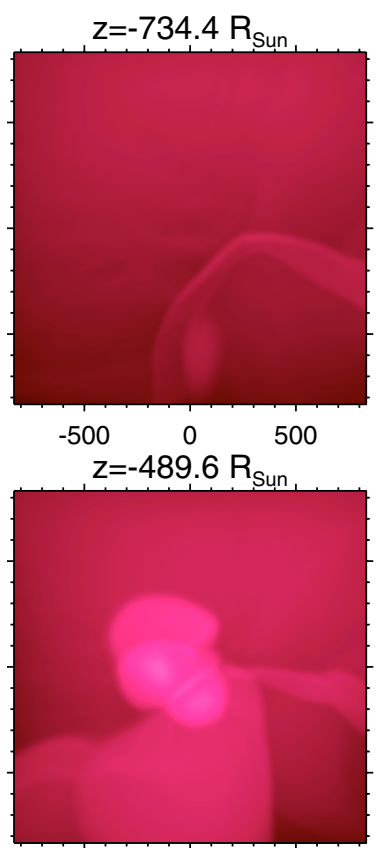

$\begin{array}{lll}-500 & 0 & 500\end{array}$

$\mathrm{Z}=-244.8 \mathrm{R}_{\text {Sun }}$

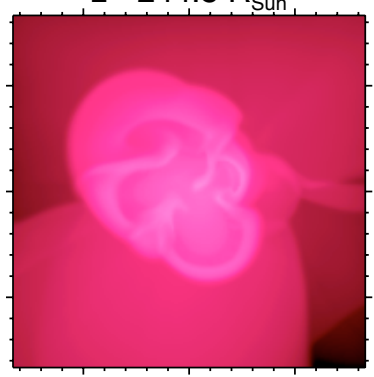

$\begin{array}{lll}-500 & 0 & 500\end{array}$ $\mathrm{z}=0.0 \mathrm{R}_{\text {Sun }}$

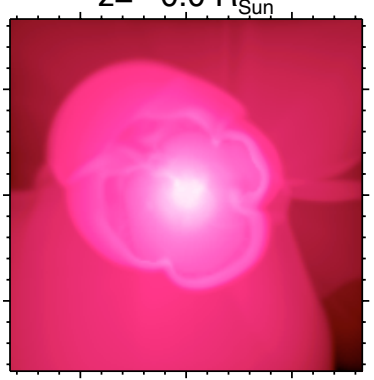

$\begin{array}{lll}-500 & 0 & 500\end{array}$ $\mathrm{Z}=244.8 \mathrm{R}_{\text {Sun }}$

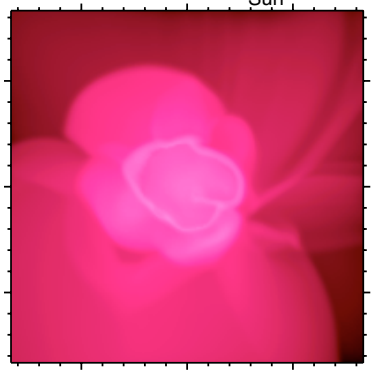

$-500 \quad 0 \quad 500$
$Z=-734.4 R_{\text {Sun }}$

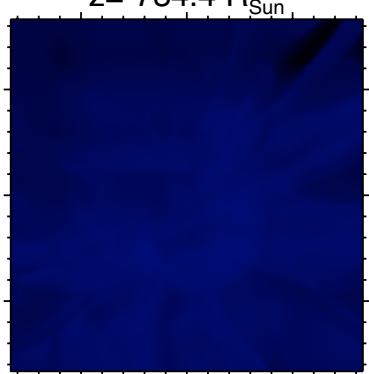

$\begin{array}{lll}-500 & 0 & 500\end{array}$

$z=-489.6 R_{\text {Sun }}$

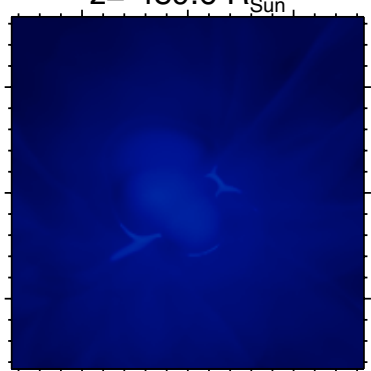

$\begin{array}{lll}-500 & 0 & 500\end{array}$ $\mathrm{Z}=-244.8 \mathrm{R}_{\text {Sun }}$

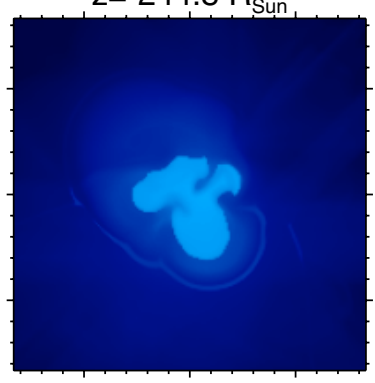

$\begin{array}{lll}-500 & 0 & 500\end{array}$ $z=0.0 R_{\text {Sun }}$
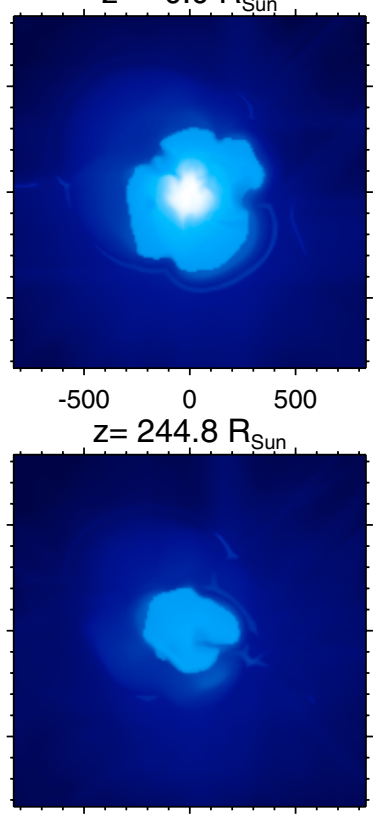

$\begin{array}{lll}-500 & 0 & 500\end{array}$
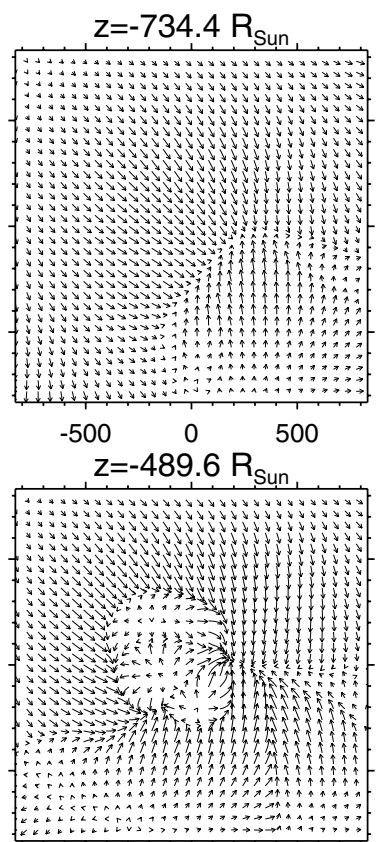

$\begin{array}{lll}-500 & 0 & 500\end{array}$

$z=-244.8 R_{\text {Sun }}$

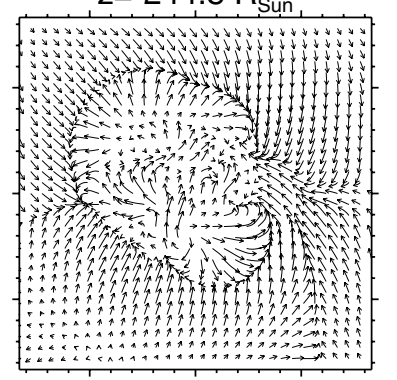

$\begin{array}{lll}-500 & 0 & 500\end{array}$

$z=0.0 R_{\text {Sun }}$

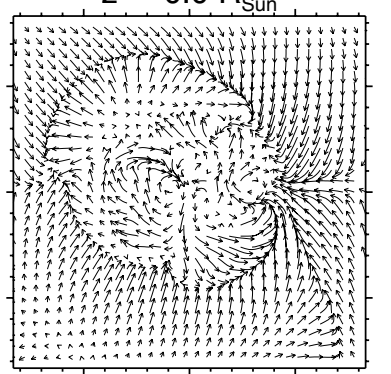

$\begin{array}{lll}-500 & 0 & 500\end{array}$

$z=244.8 R_{\text {Sun }}$

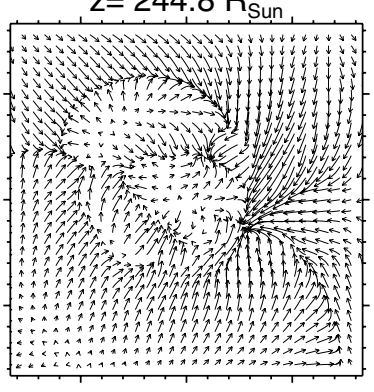

$\begin{array}{lll}-500 \quad 0 & 500\end{array}$

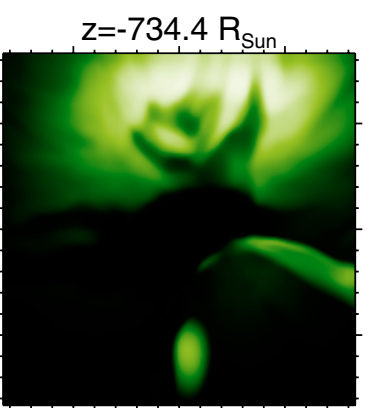

$\begin{array}{lll}-500 & 0 & 500\end{array}$

$z=-489.6 R_{\text {Sun }}$

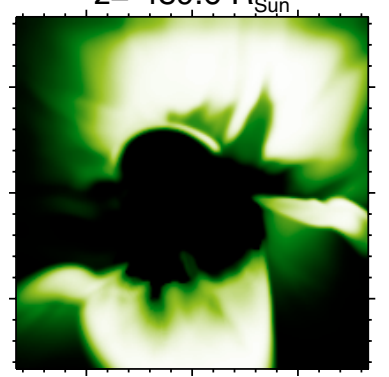

$\begin{array}{lll}-500 & 0 & 500\end{array}$

$z=-244.8 R_{\text {Sun }}$

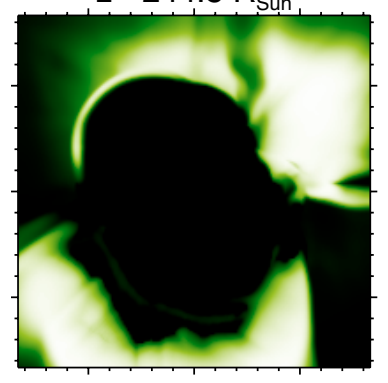

$\begin{array}{lll}-500 & 0 & 500\end{array}$ $z=0.0 R_{\text {Sun }}$
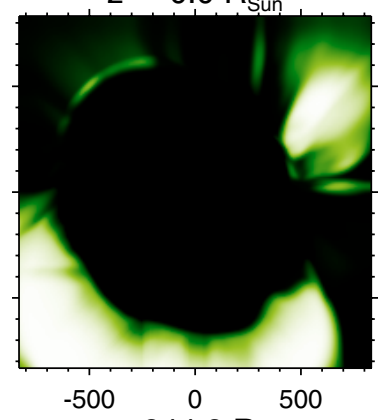

$z=244.8 R_{\text {Sun }}$

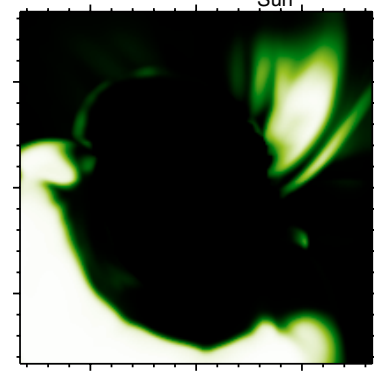

$\begin{array}{lll}-500 \quad 000 & 0\end{array}$

Fig. 3. A spatial series of slices for a selected snapshot of model $\mathrm{L}(\mathrm{st} 28 \mathrm{gm} 06 \mathrm{n} 05)$ at $t=4.06 \mathrm{yrs}$. The $z$ coordinate is indicated on top of each frame (zero corresponding to a slice through the center of the computational box). All axes are in solar radii. The displayed quantities are the same as in Fig. 2. 

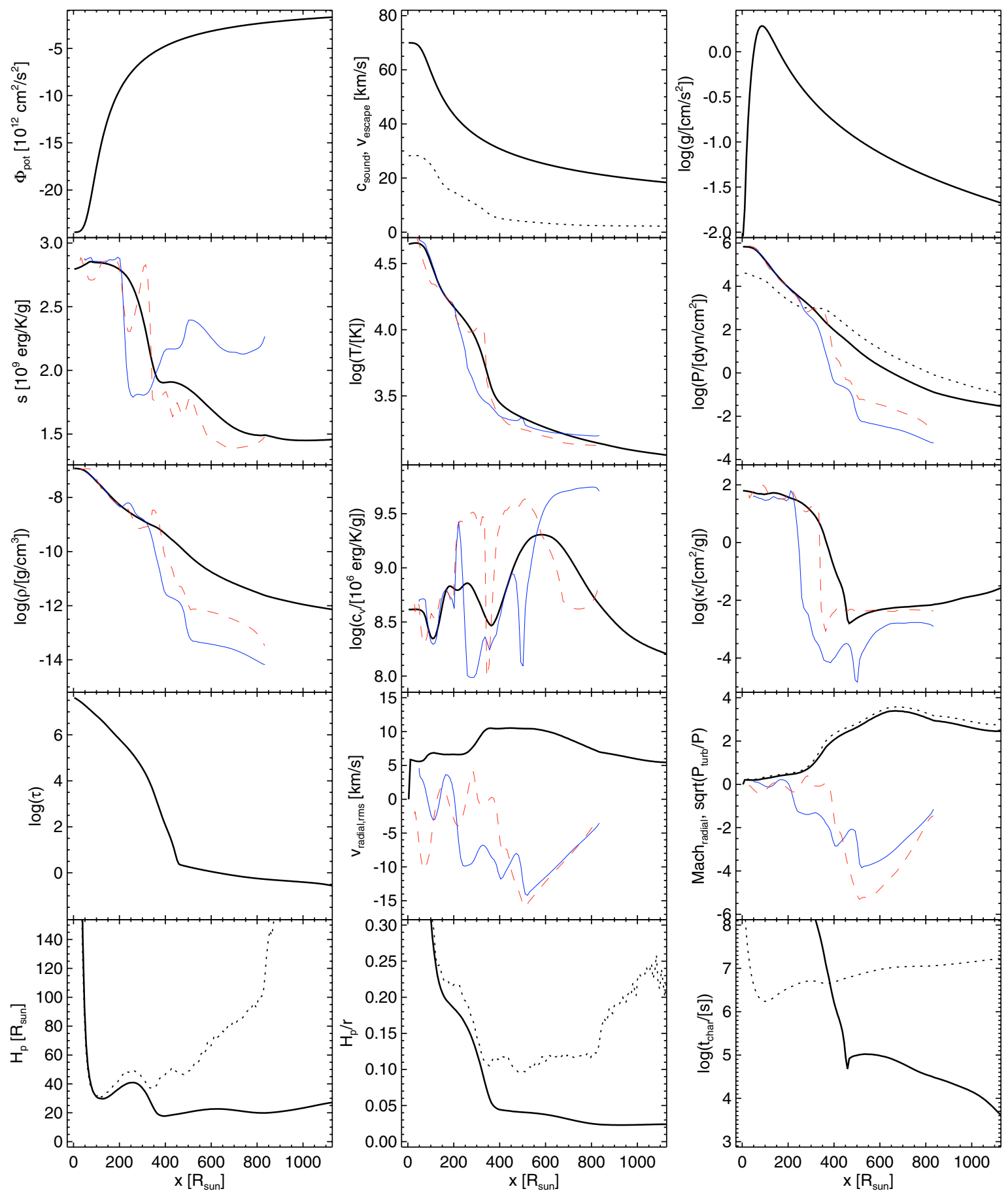

Fig. 4. Several 1D quantities over radius for model L: the thick continuous line (and in some cases the additional dotted one) refers to a quantity averaged over spherical shells and time. The two thin lines in some frames are from two selected columns at a certain instant: the thin dashed (red) line shows data in an upstream region, the thin continuous (blue) line corresponds to a downdraft. The quantities: gravitational potential $\Phi_{\text {pot }}$; escape velocity $v_{\text {escape }}$ (continuous line) and sound speed $c_{\text {sound }}$ (dotted line); acceleration due to gravity $g$; specific entropy $s$; temperature $T$; pressure $P$ with gas pressure (continuous line) and dynamical pressure $\rho v_{\text {radial }}^{2}$ (dotted line); density $\rho$; heat capacity (per volume) $c_{\mathrm{v}}$; opacity $\kappa$; optical depth from averaged quantities $\tau$; radial velocity: rms-value and two examples $v_{\text {radial }}$; radial Mach numbers "Mach" (continuous line) and square root of turbulent over gas pressure $P_{\text {turb }} / P$ (dotted line); scale height of hydrostatic and actual gas pressure, in solar radii $H_{P}$; scale height of hydrostatic and actual gas pressure, relative to distance to stellar center $H_{P} / r$; characteristic radiative and hydrodynamical time scales $t_{\text {char }}$.

\subsubsection{Atmospheric dynamics and shocks}

Going from the convective stellar interior to the thinner atmospheric layers, the character of both flow patterns and spatial structures changes significantly.

While the dynamical pressure is only a fraction of the gas pressure in the stellar interior (Fig. 4), it takes over close to the surface and dominates the total pressure in the atmosphere (note, again, that radiation pressure is not included in the 3D models, yet). The dynamic or "turbulent" pressure in the atmosphere is due to waves and shocks. Their action is visible, e.g., in the density and temperature slices in Figs. 1 and 2: small-amplitude pulsations of the stellar interior steepen up when they traverse the surface and turn into shock waves propagating into the outer 
atmosphere. Their velocities can come close to - but stay below - the escape velocity (cf. Fig. 5). The shocks cannot expand freely into empty space but are slowed down by material from previous events that has not managed to leave the gravity field of the star and that now falls back towards its surface. Therefore, the shock waves alone are not sufficient to cause efficient mass loss. The latter requires as additional mechanism acceleration due to radiation pressure on dust.

The formation, transport, and destruction of dust grains is included in model L, but without taking dust opacities and the resulting radiation pressure into account. The sequence depicted in Fig. 2 starts with a (more or less) dynamically relaxed model immediately after activating the terms that describe dust formation. The conditions in the outer atmosphere of the lower hemisphere are sufficient to allow an immediate formation of dust grains: The temperature is low enough to allow condensation and the density is high enough so that dust can form on a time scale comparable to the dynamical time scale of the atmosphere. The temperature criterion leads to a sharp inner boundary of the dust shell.

During the time span covered by Fig. 2 a shock wave starts from the stellar surface and travels into the upper left quadrant of the plots. Dissipation in the shock front leads to an increase in temperature, followed by efficient radiative cooling, restoring equilibrium. The high-temperature region is very narrow. It is followed by gas with high density and temperatures that mainly depend on the distance from the star with only small local fluctuations. Once this temperature is low enough dust formation sets in rapidly in the post-shock region (last frame in Fig. 2).

Thus, dust formation occurs predominantly in a limited range of distances from the stellar surface. Once dust grains have grown, however, they can survive as long as the temperatures remain low enough even if the densities are too small to allow further condensation. Due to the non-equilibrium nature of these dust processes, the actual dust distribution around the star depends on the history (i.e. $T(t)$ and $\rho(t)$ ) of each gas element.

Regarding the overall structure of the circumstellar dust shell it should be noted that some radial structures seen in the plots are artefacts of the simplified (speed-optimized) radiation transport scheme chosen for this model: as rays along the grid axes or diagonals do not require any interpolation they are faster to compute and were preferred over intermediate angles: steps that solve the radiation transport equation only along some axes or diagonals alternate with steps that solve the equation along rays with arbitrary directions. Therefore, a point source (for instance a hot spot on the stellar surface) does not radiate equally in all angles. Nevertheless it is clear that non-spherical structures in the density and temperature of the atmosphere due to the convection cells are reflected in the dust distribution close to the star (see Fig. 3). These inhomogeneities may provide an interesting tool for the diagnostics of AGB atmospheres and winds by combining improved 3D models with state-of-the-art interferometric observations.

\section{Extraction of pulsation properties}

The strong atmospheric shock waves associated with the giant convection cells (cf. Fig. 2) lead to a levitation of the upper atmospheric layers in the 3D models. This is comparable to the effect of stellar pulsations - simulated by a variable membrane below the photosphere (piston) - in the 1D models. In the latter case, this results in intermittent dust formation in the dense, cool wakes of the shock waves, followed by radiative acceleration of the gas-dust mixture and an outflow of material corresponding

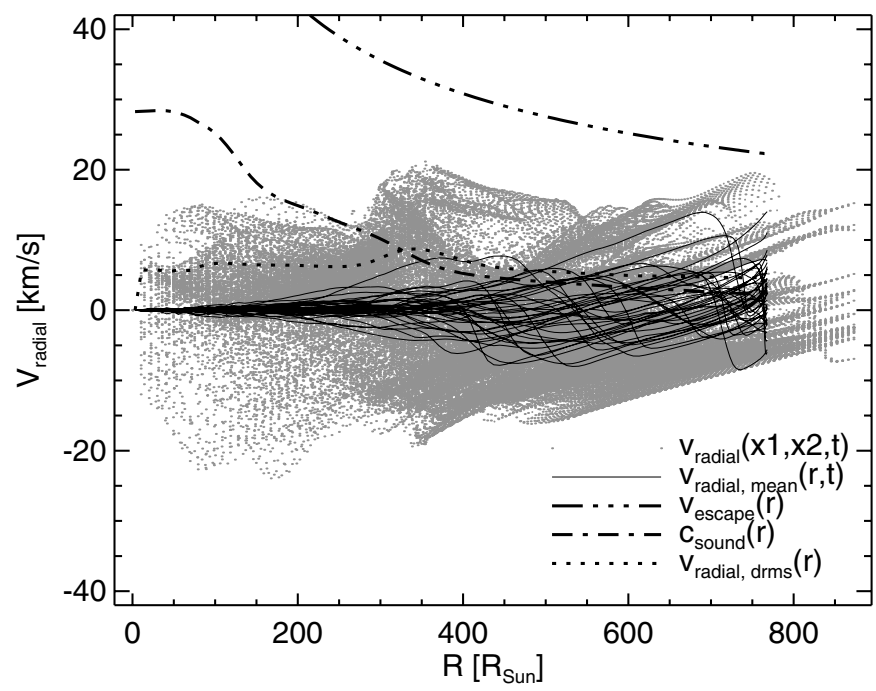

Fig. 5. Typical radial velocities (grey dots) for model $\mathrm{S}$ are of the order of $10 \mathrm{~km} \mathrm{~s}^{-1}$. Maximum values lie around $20 \mathrm{~km} \mathrm{~s}^{-1}$. Averages over spherical shells give the radial part of the velocities (black thin lines in the figure above). They account for a considerable part of the motions in the outer atmosphere, but only for a small fraction inside the convective envelope $\left(R<350 R_{\text {Sun }}\right)$. The escape velocity, time averages of the sound speed, and the rms value of the radial velocity are given as reference.

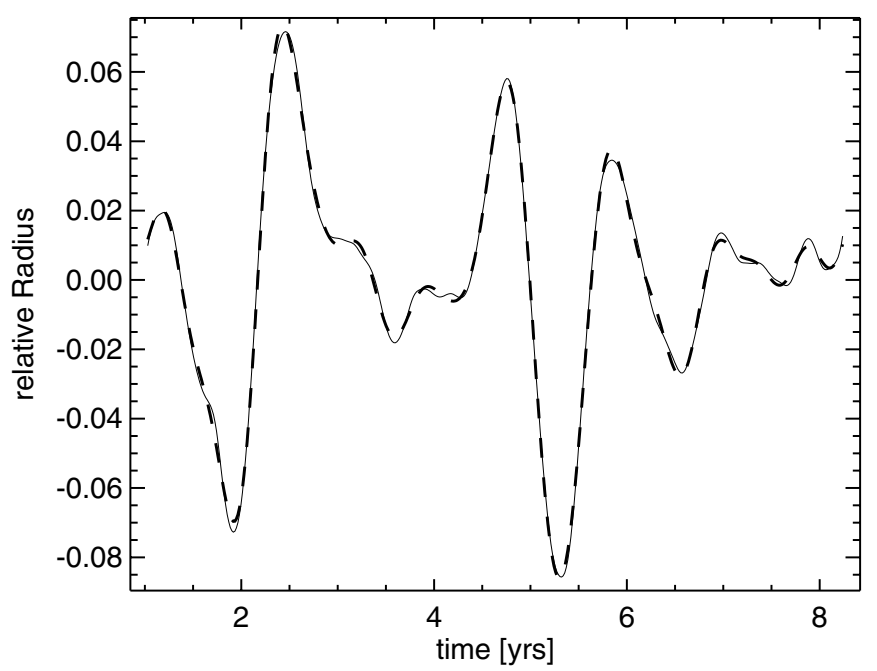

Fig. 6. Relative radius over time for model $\mathrm{S}$. The thin continuous line is derived from the $3 \mathrm{D}$ model. The thick dashed line is the fit. The average radius over the selected time interval is $R=301 R_{\odot}$. Referred to as boundary condition 3Drr01 (“3D-relative-radius-01”).

to substantial mass loss. Therefore one should - in principle expect the formation of a dust-driven wind in 3D models similar to the the outflows in spherical models. This is, however, not possible in the present version of the 3D models for two reasons.

Firstly, the star-in-a-box simulations currently contain a description of dust formation, destruction, and transport without coupling the grains to the radiation field. Consequently, the driving force of the winds, radiation pressure on dust, is not included at present. Secondly, the 3D models cover only the inner part of the atmosphere and do not span long enough time intervals to study the formation of a wind. The restriction to spherically symmetric 1D models on the other hand allows for a significantly larger computational domain and much longer time sequences, as well as making the inclusion of more detailed 


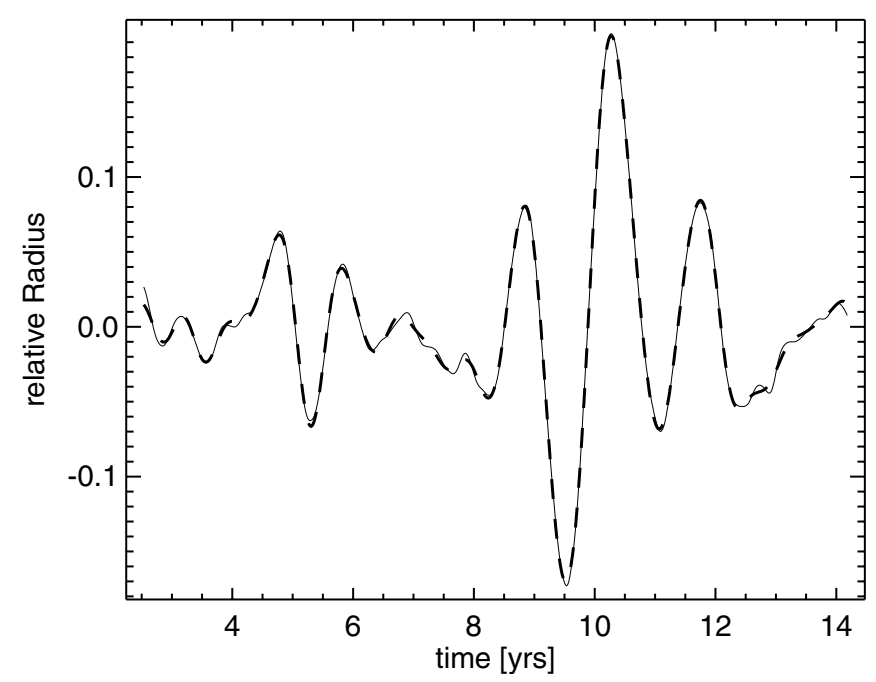

Fig. 7. Relative radius over time for the same model $\mathrm{S}$ as in Fig. 6 but a longer sequence. The thin continuous line is derived from the $3 \mathrm{D}$ model. The thick dashed line is the fit (with 20 coefficients). The average radius over the selected time interval is $R=306 R_{\odot}$. Referred to as boundary condition 3Drr03.

microphysics possible (for instance frequency-dependent radiative transfer, see Höfner et al. 2003).

To combine the advantages of both types of simulations we translate the global oscillations of the star in the 3D model into a purely radial pulsation which is described in terms of a variable radius of the subphotospheric layers. First, at given instants, we average the density of the $3 \mathrm{D}$ models over spherical shells. Integration over radius then gives the mass contained within a certain radius at a given time. Following a selected value of the integrated mass through a sequence of snapshots finally gives the radial coordinate of a mass shell over time. These variations can be used as an inner boundary for the spherically symmetric wind models, replacing the sinusoidal variations commonly used in such 1D models.

The relative radial variations of such a (hypothetical) mass shell close to the stellar surface in model $\mathrm{S}$ are plotted in Fig. 6 (continuous curve) over an interval where the first and last point match nicely. A systematic trend in the mean radius was removed by subtracting a fitted parabola. Retaining the first 14 coefficients of a Fourier decomposition gives a smooth, perfectly periodic function (dashed curve), which can be applied in the 1D models to describe the variable location of the inner piston boundary. Figure 7 shows similar data for a longer sequence and at a slightly different mean radius for the same model $\mathrm{S}$.

In the following section we compare 1D spherically symmetric wind models using these new boundary conditions derived from the 3D models with simulations based on the usual sinusoidal piston movements.

\section{1D wind models}

\subsection{Boundary conditions and modeling procedure}

The effects of large-scale convection or stellar pulsations on atmosphere and wind are simulated by applying a variable inner boundary $R_{\text {in }}(t)$ below the photosphere. The variations at this inner boundary are either extracted from 3D models as described in Sect. 4 , or are sinusoidal with a velocity amplitude $\Delta u_{\mathrm{p}}$ and a period $P$ as in previous studies (see, e.g., Höfner \& Dorfi 1997; Höfner et al. 2003). In either case, no mass flow is allowed across this inner boundary, forcing the gas close to $R_{\text {in }}$ to move with
Table 3. Boundary conditions and results of 1D wind models.

\begin{tabular}{llccc}
\hline \hline BC type & $\begin{array}{l}v_{\text {ampl }} \\
{\left[\mathrm{km} \mathrm{s}^{-1}\right]}\end{array}$ & $\begin{array}{c}\dot{M} \\
{\left[M_{\odot} / \mathrm{yr}\right]}\end{array}$ & $\begin{array}{c}v_{\text {wind }} \\
{\left[\mathrm{km} \mathrm{s}^{-1}\right]}\end{array}$ & $f_{\mathrm{c}}$ \\
\hline 3Drr01 & $\approx 3.5$ & - & - & - \\
3Drr01 $\times 1.5$ & $\approx 5$ & $1.6 \times 10^{-6}$ & 10 & 0.26 \\
3Drr03 & $\approx 5$ & $1.6 \times 10^{-6}$ & 12 & 0.24 \\
3Drr03 $\times 1.5$ & $\approx 8$ & $4.6 \times 10^{-6}$ & 11 & 0.27 \\
sinusoidal & 3.5 & $2.3 \times 10^{-7}$ & 5.5 & 0.16 \\
sinusoidal & 5.0 & $1.1 \times 10^{-6}$ & 10 & 0.20 \\
sinusoidal & 8.0 & $2.5 \times 10^{-6}$ & 10 & 0.22 \\
\hline
\end{tabular}

the boundary. The luminosity at the inner boundary varies like $L_{\text {in }}(t) \propto R_{\text {in }}^{2}(t)$, corresponding to the assumption that the total radiative flux at the inner boundary is constant.

The dynamical computation starts with a hydrostatic initial model which is characterized by the luminosity $L_{\star}$, mass $M_{\star}$, effective temperature $T_{\star}$, and the elemental abundances of the star. This initial model corresponds to the hydrostatic limit case of the radiation-hydrodynamical equations, assuming that no dust is present. In the current case, we use only one such combination of stellar parameters corresponding to the parameters of the 3D model.

In the hydrostatic initial model the outer boundary is located close to the photosphere due to the steep atmospheric density gradient. When the dynamical calculation is started, the shocks created by the pulsation and the radiation pressure on newly formed dust grains will cause the upper atmosphere to expand. At this stage the outer boundary follows the movement of the gas. If the model develops a wind, the expansion is followed up to a distance of about $20-30 R_{\star}$ where the boundary is fixed, allowing outflow. At this distance, the time-averaged wind properties (outflow velocity, degree of condensation, etc.) have usually reached their asymptotic values. In models which do not form a stellar wind the outer boundary moves along with the upper atmospheric layers during the whole computation.

In the following, we discuss two types of wind models: first we describe the results obtained with the boundary conditions extracted from the 3D simulations. Then we compare these new models to classical ones employing the usual sinusoidal piston. The wind properties of all discussed models are summarized in Table 3. It shows the type of the employed boundary condition ("3Drr" stands for "3 Dimensional relative radius"); the (max.) velocity amplitude; the mass loss rate, the mean wind velocity, and the mean degree of condensation. The boundary conditions 3Drr01 and 3Drr03 correspond to Figs. 6 and 7, respectively. The period of the sinusoidal boundary condition is 390 days.

\section{2. $1 D$ models with piston movement from $3 D$ models}

As anticipated from earlier studies of models with a sinusoidal movement of the piston, it turns out that the velocity amplitude at the inner boundary is a critical quantity. The first boundary condition derived from the 3D models, 3Drr01 (cf. Fig. 6), shows rather small relative variations of the radius coordinate, translating into maximal velocities around $3.5 \mathrm{~km} \mathrm{~s}^{-1}$. Applying this boundary condition to the 1D model results in no wind, as the levitation of the outer atmosphere and thus the dust formation is too inefficient. Increasing the variations by a factor 1.5 leads to a maximal velocity at the inner boundary of about $5 \mathrm{~km} \mathrm{~s}^{-1}$, enough to allow for dust formation and to get a pronounced stellar wind (see Table 3 for details). Such velocities are by no means unreasonable in a region just below the photosphere, as can be seen from Fig. 5 which shows radial velocities in the 3D 

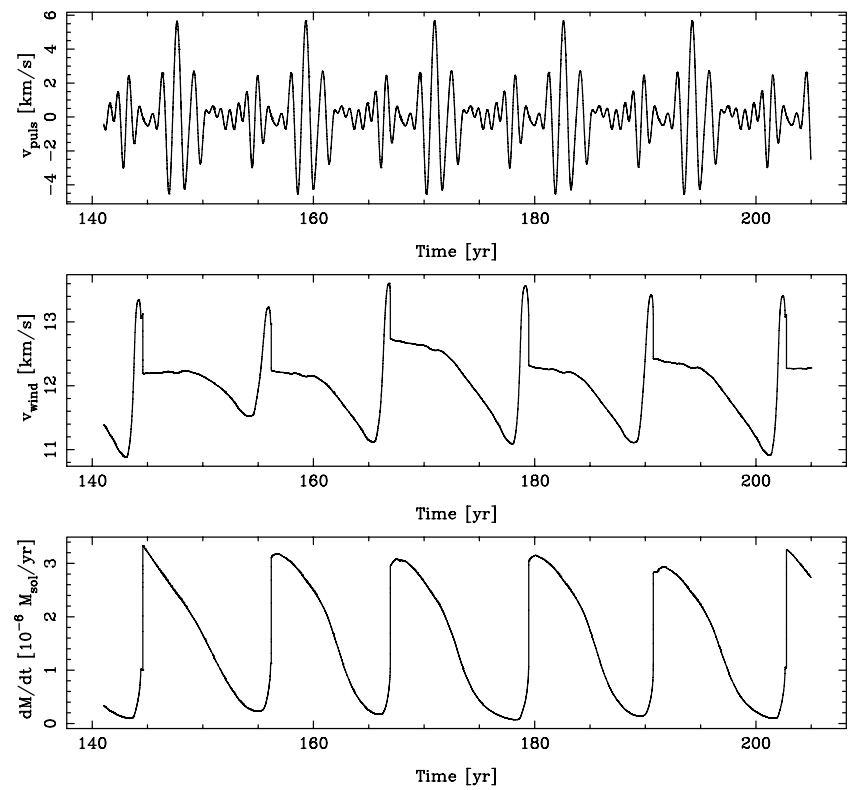

Fig. 8. Wind model with piston movement corresponding to boundary condition 3Drr03: temporal variation of selected quantities. Top panel: gas velocity at the inner boundary, just below the photosphere; middle: wind velocity at the outer boundary; bottom panel: mass loss rate.
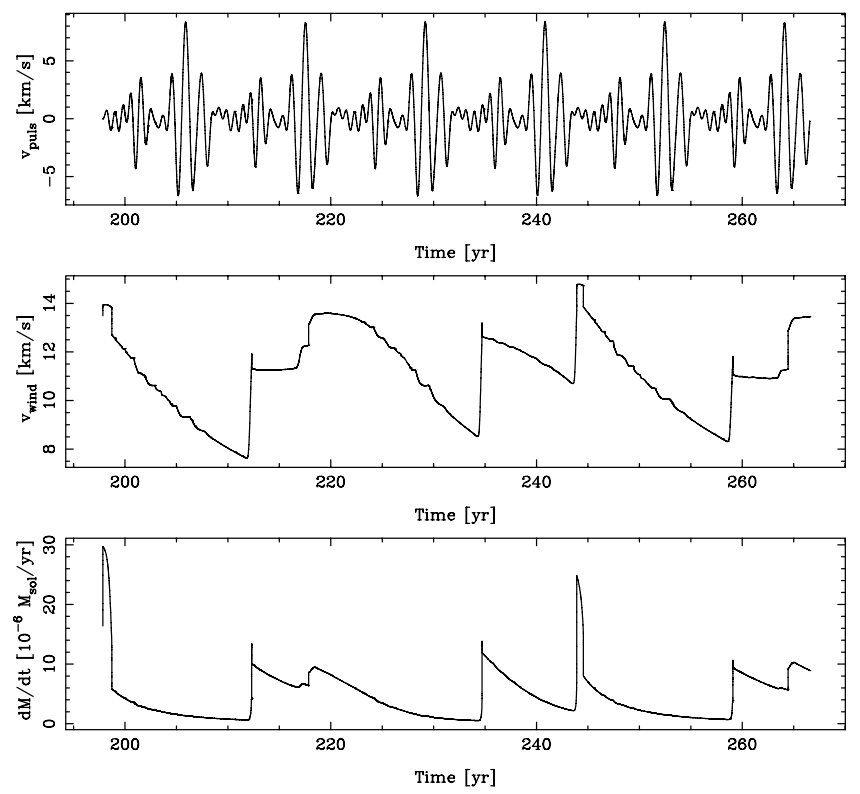

Fig. 9. Wind model with piston movement corresponding to the enhanced boundary condition 3 Drr03 $\times 1.5$. All quantities as in Fig. 8 .

model as a function of depth: the photospheric velocity fields are not exactly spherically symmetric and the spherical averages are smaller than typically encountered values.

The boundary condition 3Drr03 (cf. Fig. 7) exhibits considerably larger relative variations of the radius coordinate than 3Drr01. These variations result in maximal velocities of approximately $5 \mathrm{~km} \mathrm{~s}^{-1}$ at the inner boundary, leading to a dustdriven stellar wind quite similar to the outflow obtained with the enhanced boundary condition 3Drr01 (cf. Table 3). To illustrate the influence of the amplitude, we have also calculated a model where the variation of $3 \mathrm{Drr} 03$ was increased by a factor of 1.5 (leading to maximal boundary condition velocities around $8 \mathrm{~km} \mathrm{~s}^{-1}$ ), and obtain a wind with a mass loss rate increased by about a factor of 3 while the wind velocity and dust-to-gas ratio
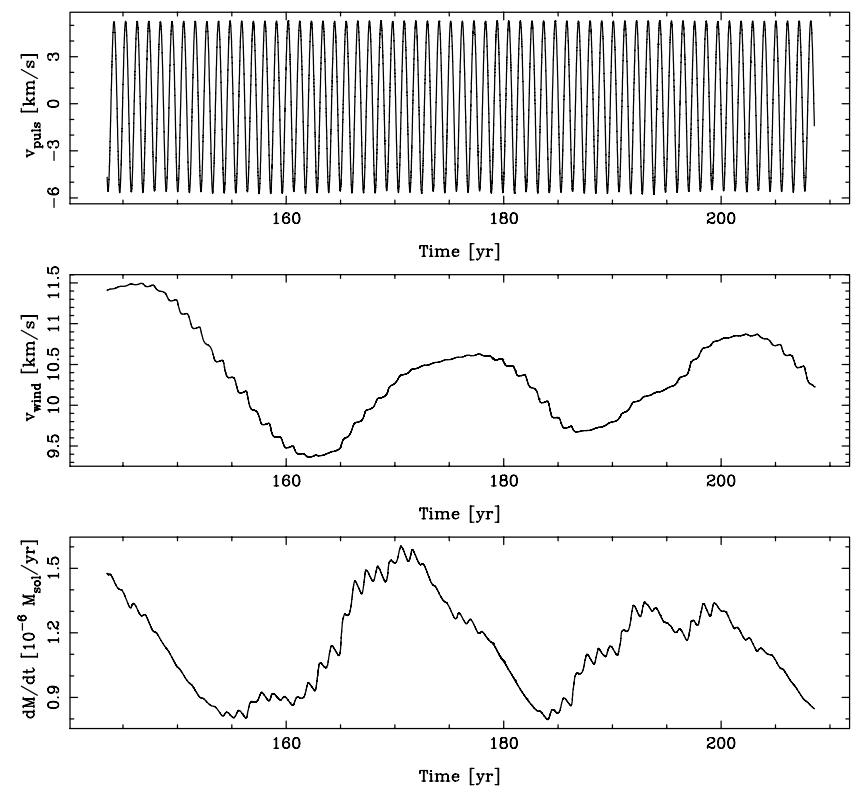

Fig. 10. Same quantities as in Fig. 8 but for a model with sinusoidal piston movement (velocity amplitude $5 \mathrm{~km} \mathrm{~s}^{-1}$ ).

remain roughly the same as with the original boundary condition 3 Drr03.

Figure 8 shows the temporal variations of selected quantities for the wind model obtained with the original boundary condition 3Drr03. Note, that the variations in the wind do not strictly follow those of at the inner boundary (piston), and that the variations of the mass loss rate are considerable. The model with the enhanced boundary condition $3 \mathrm{Drr} 03 \times 1.5$ (Fig. 9) shows much stronger irregularities and more pronounced variations of the wind properties with time, along with the higher mean mass loss rate.

\section{3. $1 D$ models with sinusoidal piston movement}

For comparison, we also show a wind model with a sinusoidally moving piston and a velocity amplitude of $5 \mathrm{~km} \mathrm{~s}^{-1}$ (cf. Fig. 10). Its amplitude is similar to the maximal variations seen in the wind model with the original boundary condition 3Drr03 (Fig. 8). The sinusoidal model with the $5 \mathrm{~km} \mathrm{~s}^{-1}$ amplitude produces a mean outflow velocity and mass loss rate comparable to the non-sinusoidal wind model (but with smaller temporal variations).

A sinusoidal model with a piston of $3.5 \mathrm{~km} \mathrm{~s}^{-1}$ (which is probably closer to the average piston velocity in the nonsinusoidal case 3Drr03) is found to have a much lower mass loss rate and wind velocity. Nevertheless the sinusoidal model with a piston amplitude of $3.5 \mathrm{~km} \mathrm{~s}^{-1}$ does produce an outflow, in contrast to the non-sinusoidal model with boundary condition 3Drr01. The latter model has a similar maximal piston velocity which is, however, only reached about 2 times during an interval of approximately 7 years - as opposed to a period of about one year in the sinusoidal model - and therefore not sufficient to trigger a dust-driven wind as mentioned above.

In Table 3 we also list a sinusoidal model with an amplitude of $8 \mathrm{~km} \mathrm{~s}^{-1}$, for comparison with the model 3 Drr03 $\times 1.5$ which has a comparable maximal piston velocity amplitude. The ratio of the mass loss rates of the two sinusoidal models with 8 and $5 \mathrm{~km} \mathrm{~s}^{-1}$ turns out to be somewhat smaller than the corresponding ratio for the two non-sinusoidal models. 


\section{Conclusions}

We have presented a first set of 3D radiation hydrodynamics simulations of the outer convective layers and the inner atmosphere of a tip-AGB star, including non-local radiation transport and detailed microphysics (equation of state and opacities). We have analyzed both the properties of the large-scale convective flows, as well as similarities and differences in the atmospheric layers compared to spherically symmetric models.

The surface of the model star is dominated by a few giant convection cells. Their size is roughly consistent with that of solar granules scaled with the pressure scale height, confirming the arguments of Schwarzschild (1975). The model shows irregular pulsations that interact with the convective cells and trigger shock waves in the thin photospheric layers, leading to a dynamical levitation of the outer atmosphere that agrees well with earlier 1D simulations of atmospheres and winds. Dust formation occurs in dense, cool regions above the stellar photosphere where gas has been compressed by propagating shock waves and subsequently cooled efficiently by radiative processes. The time scales for dust formation are comparable to the hydro- and thermodynamical time scales in the atmosphere leading to nonequilibrium effects, such as incomplete condensation and intricate patterns reflecting the inhomogeneities in the gas. A timedependent description of the dust component is necessary, as also indicated by 1D models with more detailed microphysics.

While the 3D models - in contrast to their 1D counterparts - contain the top of the convection zone including the layers where the pulsations are excited, they cover only the very innermost fraction of the stellar envelope. The models are large enough to demonstrate the formation of dust which usually occurs at a distance of 1-2 stellar radii from the surface, but cannot follow the acceleration of the dust grains all the way out of the stellar gravitational potential, which is possible to simulate in 1D models. In addition, the 3D models have a relatively low (constant) spatial resolution, grey opacities, and comparatively short time sequences. Still they are time-consuming and require a few CPU-years on an 8 processor $900 \mathrm{MHz}$ SunFire machine - in contrast to several CPU-hours per 1D model.

To combine the strengths of both types of models and study dust-driven winds in connection with atmospheric dynamics typical of the 3D "star-in-a-box" simulations, we averaged the 3D models over spherical shells and derived a description for the motion of the piston at the lower boundary of the 1D models. We also experimented with scaling up the deduced average velocity amplitude to account for non-spherical velocity fields. The 1D runs employing this piston motion demonstrate that the 3D simulations give radial velocities of the right amplitude to produce a dust-driven stellar wind. Furthermore, we demonstrate that in case of a non-sinusoidally oscillating piston the mass loss rates are mainly determined by the maximum velocities of the piston.

Our goal for the far future is to perform all these simulations in 3D. However, time-dependent three-dimensional models require an enormous amount of computer resources and therefore have to adopt simplifications that are not necessary in onedimensional calculations. We plan gradual improvements of the 3D models in terms of numerical resolution, extent into the circumstellar envelope, time span covered by a simulation, number of frequency bins, and coupling of the dust to the radiation field including radiation pressure onto dust. 3D and 1D models will continue to coexist for quite some time.

Acknowledgements. This project is partially supported by the Swedish Research Council (Vetenskapsrådet) and by a stipend financed by the Schönberg donation. We thank Kjell Eriksson and Bengt Gustafsson for their helpful comments about the manuscript. The computations were performed on UPPMAX resources under Project p2003019.

\section{Appendix A: Details of the 3D simulations}

\section{A.1. Setup of the 3D models}

The obvious way to construct a start model for a 3D simulation is to map a 1D stellar structure model onto the 3D grid. However, this procedure bears some difficulties: First, one needs a snapshot from a stellar evolution track for exactly the right set of stellar parameters. And 1D and 3D models have to use the same set of microphysics (equation of state, opacities) and boundary conditions. The latter is a problem for the deep stellar interior because this region is represented only very crudely in the 3D case: the gravitational potential and the region where energy is produced is smoothed over a fraction of the stellar radius (about $20 \%: r_{0}=78 R_{\odot}$ ). This seems justified because simulations of the solar convection show that the very surface layers where the granules reside are most important for the dynamics of the layers below. In the outer layers the gravitational potential is described by a simple spherical $1 / r$ profile.

A severe difference between 1D and 3D cases lies in the description of the stellar surface where 1D stellar structure models usually rely on simple 1D atmosphere models, that treat convection in the framework of the mixing-length theory. The $3 \mathrm{D}$ simulations on the other hand are able to model these layers in great detail and automatically include, e.g., dynamical pressure and spatial convective inhomogeneities in the temperature, density, and velocity fields. The actual outer boundaries of the 3D model are transmitting for fluid motions and radiation.

To get a start model, first a 1D stellar structure model was constructed that was then mapped onto a spherical 3D grid adding small amounts (on the order of $10 \mathrm{~m} \mathrm{~s}^{-1}$ ) of random velocities. For the 1D model hydrostatic equilibrium was assumed overall. The outer layers were constructed assuming a grey plane-parallel $T(\tau)$ relation. In the interior the entropy was limited to a certain value to enforce an almost adiabatic stratification due to convection. The entropy value was just a guess and turned out to be somewhat too large.

Just after starting the simulation (model st28gm06n01; cf. Table 2) there is no convective energy transport yet. However, in the artificially enlarged stellar core $7000 L_{\odot}$ are injected, which leads to a temperature rise in the center and an expansion of the model. At the surface radiative energy losses without sufficient convective supply from the interior lead to a cooling of the photospheric layers. In the superadiabatic sub-photospheric layers convective cells arise. They turn later from a symmetric (due to the numerical grid) small-scale pattern into global symmetric and (after about 3.5 stellar years) asymmetric cells. These cool the interior efficiently and cause a shrinking of the stellar radius that varies significantly in time. Even later, a stationary dipole flow evolves that sends material through the artificial stellar core. The mean entropy of the material in the interior is too low, and the stellar radius turns out to be smaller than intended.

Therefore, a snapshot from the middle of this sequence was taken with the densities multiplied by a factor 1.2 and the the velocities reduced by multiplying with a factor 0.7 to start the run st28gm06n02. This procedure was repeated a few times in the early phase of the simulation until the average radius reached the intended value. To prevent the matter from taking the "shortcut" through the artificial stellar core, a strong drag force was added within a radius of $r_{0}=78 R_{\odot}$. After some time this run seemed to relax to a stationary steady state. Results are shown in 
Fig. 1, and the derived mean radius variations are used as a variable inner boundary in the 1D wind models (see Sects. 4 and 5).

A snapshot from the latter simulation with added grid points to allow a better coverage of the extended atmosphere was used as starting point for run st28gm06n03.

The run st28gm06n05 includes terms that describe the formation and transport of dust with with a carbon to oxygen ratio of $\mathrm{C} / \mathrm{O}=1.4$. This run crashed at a later stage because at some instant the temperature in one corner of the computational box dropped below the values contained in the tabulated equation of state. This equation of state table was extended towards lower temperatures and the simulation was restarted with a snapshot shortly before the crash to perform run st28gm06n06. Consecutive snapshots from runs st28gm06n05 and st28gm06n06 were combined into the large model " $\mathrm{L}$ ".

The first experimental model including dust, called st28gm06n04 (not shown in Table 2), had quite a high $\mathrm{C} / \mathrm{O}$ ratio (1.8) and formed dust very quickly. In the following run (st28gm06n05) the $\mathrm{C} / \mathrm{O}$ ratio was reduced to the more representative value of 1.4 , as described above.

\section{A.2. Physical and numerical challenges}

Compared to existing 3D radiation hydrodynamics simulations of solar granulation the models of an AGB star presented in this paper are far less mature. However, there are several factors that make simulations of an AGB star more difficult (in terms of code stability and CPU time) than comparable simulations of parts of the solar surface:

Even in the solar case the solution of the radiation transport takes a significant fraction of the total CPU time but for the AGB star the ratio of the radiative to the hydrodynamical time step is about a factor 100 smaller. As a result, the CPU time necessary to compute, e.g., the sound-crossing time through the computational volume (or a convective turnover time) is significantly increased for an AGB star model. This is the main reason why the current models use simple grey opacity tables and still have only a moderate spatial resolution (up to $171^{3}$ grid points).

Violent convective flows and shocks with large Mach numbers should cause no problem for the hydrodynamics scheme alone. However, in connection with the temperature sensitivity of opacity (Fig. 4) the resulting possible extreme variations in optical thickness from grid point to grid point tend to cause problems for the radiation transport scheme and require a very stable radiation transport operator. In addition, this operator has to deal with the strong, badly resolved sub-photospheric jump in temperature due to the increase in opacity. Numerical problems can arise from cross-talk between grid cells due to inappropriate interpolations of the source function: the radiative cooling of a hot cell might not be restricted to that cell but can "leak" into its neighbors. The radiation transport operator tries to minimize these interpolation errors.

Waves (pulsations in the stellar interior and shocks in the atmosphere) and global convective flows lead to a stronger coupling of the deep interior and the outer atmosphere to the stellar surface than in the solar case. As a consequence, AGB star models should not only cover the surface layers but should reach as deeply into the star and as far out into the atmosphere as possible - with proper modeling of the relevant physical processes, of course.

\section{References}

Asida, S. M. 2000, ApJ, 528, 896

Asida, S. M., \& Tuchman, Y. 1997, ApJ, 491, L47

Asplund, M., Ludwig, H.-G., Nordlund, A., \& Stein, R. F. 2000, A\&A, 359, 669

Aufdenberg, J. P., Ludwig, H.-G., \& Kervella, P. 2005, ApJ, 633, 424

Bowen, G. H. 1988, ApJ, 329, 299

Collet, R., Asplund, M., \& Trampedach, R. 2006, ApJ, 644, L121

Dorfi, E. A., \& Drury, L. O. C. 1987, J. Comput. Phys., 69, 175

Fleischer, A. J., Gauger, A., \& Sedlmayr, E. 1992, A\&A, 266, 321

Freytag, B., \& Salaris, M. 1999, ApJ, 513, L49

Freytag, B., Steffen, M., \& Dorch, B. 2002, Astron. Nachr., 323, 213

Freytag, B., Steffen, M., Wedemeyer-Böhm, S., \& Ludwig, H.-G. 2004, CO5BOLD User Manual,

http://www.astro.uu.se/ bf/co5bold_main.html

Gadun, A. S., Hanslmeier, A., Pikalov, K. N., et al. 2000, A\&AS, 146, 267

Gail, H.-P., \& Sedlmayr, E. 1988, A\&A, 206, 153

Gauger, A., Sedlmayr, E., \& Gail, H.-P. 1990, A\&A, 235, 345

Gautschy-Loidl, R., Höfner, S., Jørgensen, U. G., \& Hron, J. 2004, A\&A, 422, 289

Hauschildt, P. H., Baron, E., \& Allard, F. 1997, ApJ, 483, 390

Höfner, S. 2005, in Proceedings of the 13th Cambridge Workshop on Cool Stars, Stellar Systems and the Sun, ESA SP-560, ed. F. Favata, et al., 335

Höfner, S. 2007, in Why Galaxies Care About AGB Stars, ed. F. Kerschbaum, et al., ASP. Conf. Ser., 378, 145

Höfner, S., \& Andersen, A. C. 2007, A\&A, 465, L39

Höfner, S., \& Dorfi, E. A. 1997, A\&A, 319, 648

Höfner, S., Gautschy-Loidl, R., Aringer, B., \& Jørgensen, U. G. 2003, A\&A, 399, 589

Iglesias, C. A., Rogers, F. J., \& Wilson, B. G. 1992, ApJ, 397, 717

Jacobs, M. L., Porter, D. H., \& Woodward, P. R. 1998, BAAS, 30, 1316

Jeong, K. S., Winters, J. M., Le Bertre, T., \& Sedlmayr, E. 2003, A\&A, 407, 191

Lattanzio, J. C. \& Wood, P. 2004, in Asymptotic Giant Branch Stars, ed. H. J. Habing, \& H. Olofsson (Springer), 23

Nordlund, А. 1982, A\&A, 107, 1

Nowotny, W., Aringer, B., Höfner, S., Gautschy-Loidl, R., \& Windsteig, W. 2005a, A\&A, 437, 273

Nowotny, W., Lebzelter, T., Hron, J., \& Höfner, S. 2005b, A\&A, 437, 285

Robinson, F. J., Demarque, P., Li, L. H., et al. 2003, MNRAS, 340, 923

Robinson, F. J., Demarque, P., Li, L. H., et al. 2004, MNRAS, 347, 1208

Rouleau, F., \& Martin, P. G. 1991, ApJ, 377, 526

Schwarzschild, M. 1975, ApJ, 195, 137

Skartlien, R., Stein, R. F., \& Nordlund, Å, 2000, ApJ, 541, 468

Steffen, M., Ludwig, H.-G., \& Krüß, A. 1989, A\&A, 213, 371

Stein, R. F., \& Nordlund, A. 2000, Sol. Phys., 192, 91

van Leer, B. 1977, J. Comp. Phys., 23, 276

Vögler, A. 2004, A\&A, 421, 755

Wedemeyer, S., Freytag, B., Steffen, M., Ludwig, H.-G., \& Holweger, H. 2004, A\&A, 414, 1121

Wedemeyer-Böhm, S., Kamp, I., Bruls, J., \& Freytag, B. 2005, A\&A, 438, 1043 Willson, L. A. 2000, ARA\&A, 38, 573

Woitke, P. 2003, in Modelling of Stellar Atmospheres, ed. N. Piskunov, W. W. Weiss, \& D. F. Gray, IAU Symp., 210, 387

Woitke, P. 2006a, A\&A, 452, 537

Woitke, P. 2006b, A\&A, 460, L9

Wood, P. R. 1979, ApJ, 227, 220

Woodward, P. R., Porter, D. H., \& Jacobs, M. 2003, in 3D Stellar Evolution, ASP Conf. Ser., 293, 45 